\title{
A SIMPLE NONLINEAR DYNAMIC STABILITY PROBLEM ${ }^{1}$
}

BY B. J. MATKOWSKY

Communicated by Fred Brauer, October 29, 1969

In this paper we consider a relatively simple problem to illustrate a method for treating nonlinear dynamic stability problems. This method is believed to be the first to treat arbitrary, though small, initial perturbations. Specifically, we consider

$$
-u_{t}+u_{x x}=\lambda f(u) \text { for } 0<x<\pi, \quad t>0
$$

subject to the boundary conditions

$$
u=0 \quad \text { at } \quad x=0, \pi
$$

and the initial condition

$$
u(x, 0 ; \epsilon)=h(x ; \epsilon) \sim \epsilon h(x) .
$$

Here the symbol $\sim$ denotes asymptotic equivalence, and $\epsilon$ is a small parameter to be defined below. We assume that $f(u)$ can be expanded in a Taylor series in $u$ about $u=0$ with $f(0)=0 f^{\prime}(0)<0, f^{\prime \prime}(0)=0$, $f^{\prime \prime \prime}(0)>0$, and that $h(0)=h(\pi)=0$.

This problem is as a mathematical model for the temperature distribution in a bar with a nonlinear heat source of magnitude $-\lambda f(u)$, on the boundary of which the temperature is prescribed to be zero. We wish to study the stability of the equilibrium temperature distribution $u_{0} \equiv 0$. To do so, we must determine whether it can sustain itself against perturbations (to which all physical systems are subjected). That is, we must see whether all perturbations decay to zero, or whether some perturbations grow (perhaps into new stationary (time independent) solutions of (1)-(2)). Therefore we study the time development of the solution with initial condition $h(x)$ representing the perturbation. The stability of solutions $u_{0} \neq 0$, and satisfying nonhomogeneous boundary conditions, is treated in similar fashion by considering the problem for $\bar{u}=u-u_{0}$.

AMS Subject Classifications. Primary 3507, 3513, 3514, 3515, 3516, 3536.

Key Words and Phrases. Nonlinear partial differential equations, dynamic stability, formal asymptotic expansion.

1 Another version [1] of the method presented in this paper, was delivered at the S.I.A.M. Conference on Qualitative Theory of Differential and Integral Equations held at Madison, Wisconsin in August 1968. 
The time independent form of (1)-(2) is a nonlinear eigenvalue problem

$$
\begin{aligned}
& v_{x x}=\lambda f(v) \quad 0<x<\pi \\
& v=0 \quad \text { at } \quad x=0, \pi
\end{aligned}
$$

whose solutions are stationary or steady state solutions of (1)-(2). It can be shown that the solution $u_{0}=0$ is stable if $\lambda$ is less than a critical value $\lambda_{c}$, and unstable for $\lambda$ greater than $\lambda_{c}$. This is related to the fact that for $\lambda\left\langle\lambda_{c}, v=0\right.$ is the unique solution of (4)-(5), and that a new solution of (3)-(4) branches off or bifurcates from $v=0$ at $\lambda=\lambda_{c}$. We shall examine the post critical $\left(\lambda>\lambda_{c}\right)$ behavior of $u$ in a neighborhood of $\lambda=\lambda_{c}$. That is, for $\lambda>\lambda_{c}$, we consider the evolution in time of an initial perturbation of $u_{0}=0$. In particular we wish to determine whether the perturbation grows into the nontrivial solution (4)-(5). We are interested in the dependence of the solution $u$ on the parameter $\lambda$ in a neighborhood of $\lambda=\lambda_{c}$, and derive a formal asymptotic representation for $u$ in powers of a small parameter $\epsilon$, related to $\lambda-\lambda_{c}$ (alternatively, a norm of $u$ ). Our method provides for the systematic determination of all the terms in that expansion and is presumably valid uniformly for all time. When specialized to the steady state, our results are in agreement with those obtained by Millman and Keller [3], who used a parametric expansion to study the time independent problem.

The essential feature of the theory is the scaling of the time variable. To find the asymptotic expansion of the solution, any of the standard techniques may be used e.g., stretching and matching, two timing, multiple timing, etc. In this paper we employ the two timing technique, while in another paper [1], in which we consider a model problem arising in Fluid Dynamics, we use the stretching and matching technique of boundary layer theory to derive inner, outer and uniform expansions. In some problems (e.g., [1]) there is freedom of choice in the procedure to be adopted, while in others, the procedure is dictated by the nature of the problem (see e.g., Reiss and Matkowsky [2], for a problem where the matching technique cannot be used).

As a first attempt at solving the problem (1)-(3), we neglect the nonlinear part of $f(u)$ in (1), and consider the resulting linear problem. This analysis may be thought of as having validity for infinitesimal perturbations, and for small time. Thus linearizing (1) we obtain

$$
-u_{t}+u_{x x}-\lambda f^{\prime}(0) u=0 \text { for } 0<x<\pi, \quad t>0 .
$$


The solution of (6), (2), and (3) is

$$
u(x, t ; \epsilon) \sim \epsilon \sum_{n=1} h_{n} e^{\sigma_{n} t} \sin n x+O\left(\epsilon^{2}\right)
$$

where

$$
\sigma_{n}=\sigma_{n}(\lambda)=-f^{\prime}(0)\left(\lambda-\lambda_{n}\right)
$$

with

$$
\lambda_{n}=-n^{2} / f^{\prime}(0),
$$

and $h_{n}$ are determined from the Fourier representation

$$
h(x)=\sum_{n=1} h_{n} \sin n x .
$$

Now $u_{0}=0$ is stable or unstable according as $\sigma \lessgtr 0$. Thus, the threshold of instability occurs for the smallest $\lambda$ such that $\sigma=0$, so that

$$
\lambda_{c} \equiv \min \lambda_{n}=-1 / f^{\prime}(0) .
$$

We note that for $\lambda<\lambda_{c}$, all the $\sigma_{n}$ are negative, and the perturbation decays to zero. If $\lambda$ is slightly greater than $\lambda_{c}, \sigma_{1}>0$, but all the other $\sigma_{n}<0$. Thus the linear theory predicts stability for $\lambda<\lambda_{c}$, and instability for $\lambda>\lambda_{c}$. For $\lambda>\lambda_{c}$, the perturbation will grow exponentially in time with a growth rate proportional to $\lambda-\lambda_{c}$. Clearly, this exponentially growing "solution" according to the linear theory, cannot represent the actual solution for very long, for it will soon grow sufficiently large so that the nonlinear terms become important. Then a nonlinear analysis becomes necessary.

In our nonlinear analysis, we shall consider three relevant time regions. The first, which we denote by $t$, is an initial time region, in which the solution begins to develop from the initial perturbation. Here we expect the linear theory to be valid. The second region, denoted by $\tau$, is one in which nonlinear effects become important, and the solution continues to evolve in time until it approaches the steady state which is the third region, reached in the limit as $\tau$ becomes infinite.

Since we are examining the stability of $u_{0}=0$ in a neighborhood of $\lambda=\lambda_{c}$, it is natural to expand about these quantities. Therefore, we define $\epsilon$ by the relationship

$$
\epsilon^{2} \equiv \lambda-\lambda_{c} .
$$

The parameter $\epsilon$ is thus a measure of the nearness of $\lambda$ to $\lambda_{c}$. We introduce the variable $\tau$ by setting 


$$
\tau=\epsilon^{2} t
$$

and assume that

$$
u(x, t ; \epsilon) \sim \sum_{j=1} w^{j}(x, t, \tau) \epsilon^{j}
$$

where each $w^{i}$ is a bounded function of each of its arguments. Inserting (12), (13) and (14) into (1), and setting the coefficient of each power of $\epsilon$ to zero, we obtain a recursive system of equations for the determination of the functions $w^{j}$.

$$
\begin{aligned}
& L_{\lambda_{c} w^{1}} \equiv-w_{t}^{1}+w_{x x}^{1}-\lambda_{c} f^{\prime}(0) w^{1}=0, \\
& L_{\lambda_{c} w^{2}}=0, \\
& L_{\lambda_{c}} w^{3}=w_{\tau}^{1}+\left(\lambda_{c} f^{\prime \prime \prime}(0) / 6\right)\left(w^{1}\right)^{3}+f^{\prime}(0) w^{1}
\end{aligned}
$$

or in general

$$
L_{\lambda_{c}} w^{j}=r_{j}\left(w^{1}, \cdots, w^{j-2}\right) .
$$

A general expression for the right-hand side $r_{j}$ of (18) can be written down, though we do not do so here. In a similar manner, by employing (14) in (2) and (3) we obtain the boundary and initial conditions for this system as

$$
w^{j}=0 \quad \text { at } x=0, \pi \quad(j=1,2,3, \cdots)
$$

and

$$
\begin{aligned}
& w^{1}(x, 0)=h(x), \\
& w^{j}(x, 0)=0 \quad(j>1) .
\end{aligned}
$$

Equations (17) and (18) are inhomogeneous forms of (15). Since (15) subject to (19) with $j=1$ possesses nontrivial solutions, a necessary condition for bounded solutions of (17) and (18) to exist is that their right-hand sides satisfy the orthogonality relations

$$
\left(w^{1}, r_{k}\right) \equiv \lim _{T \rightarrow \infty} \frac{1}{T} \int_{0}^{T} \int_{0}^{\pi} w^{1} r_{k} d x d t=0 \quad(k=2,3,4, \cdots) .
$$

The solution $w^{1}$ of (15) and (19) with $j=1$, is given by

$$
w^{1}=\sum_{n=1} A_{n}^{1}(\tau) e^{\sigma_{n}\left(\lambda_{c}\right) t} \sin n x,
$$

where the functions $A_{n}^{1}(\tau)$ are as yet undetermined. Using the result that 


$$
\sigma_{1}\left(\lambda_{c}\right)=0,
$$

we write (22) as

$$
w^{1}=A_{1}^{1}(\tau) \sin x+\sum_{n=2} A_{n}^{1}(\tau) e^{\sigma_{n}\left(\lambda_{c}\right) t} \sin n x .
$$

We note that

$$
\sigma_{n}\left(\lambda_{c}\right)<0 \quad(n=2,3, \cdots)
$$

so that all the terms occurring in $\sum$ in (24), decay exponentially in time. Similarly, the solution $w^{2}$ of (16) and (19) with $j=2$ is

$$
w^{2}=A_{1}^{2}(\tau) \sin x+\sum_{n=2} A_{n}^{2}(\tau) e^{\sigma_{n}\left(\lambda_{c}\right) t} \sin n x .
$$

The orthogonality relation (21) with $k=3$, implies that

$$
\dot{A}_{1}^{1}+f^{\prime}(0) A_{1}^{1}+\left(\lambda_{c} f^{\prime \prime \prime}(0) / 8\right)\left(A_{1}^{1}\right)^{3}=0 .
$$

Here denotes differentiation with respect to $\tau$. The solution of this first order nonlinear ordinary differential equation for the amplitude function $A_{1}^{1}(\tau)$ is

$$
A_{1}^{1}(\tau)= \pm\left(\frac{-8 f^{\prime}(0)}{8 c+\lambda_{c} f^{\prime \prime \prime}(0) e^{-2 f^{\prime}(0) \tau}}\right)^{1 / 2} e^{-f^{\prime}(0) \tau}
$$

or using (11), we have that

$$
A_{1}^{1}(\tau)= \pm\left(\frac{8\left(f^{\prime}(0)\right)^{2}}{f^{\prime \prime \prime}(0) e^{-2 f^{\prime}(0) \tau}-8 c f^{\prime}(0)}\right)^{1 / 2} e^{-f^{\prime}(0) \tau}
$$

The constant $c$ is determined by the initial condition for $A_{1}^{1}(0)$, which by (20a) and (10) is

$$
A_{1}^{1}(0)=h_{1} .
$$

Thus $c$ is given by

$$
c=f^{\prime \prime \prime}(0) /\left(8 f^{\prime}(0)\right)-f^{\prime}(0) /\left(h_{1}\right)^{2} .
$$

We note that as $\tau$ becomes infinite, $A_{1}^{1}(\tau)$ approaches the constant $-\left(h_{1} /\left|h_{1}\right|\right)\left(8 / f^{\prime \prime \prime}(0)\right)^{1 / 2} f^{\prime}(0)$, so that the leading term of the expansion describes a stationary state, whose dependence on the initial data is one of sign only. Higher order terms $w^{j}$ in the expansion can be determined similarly. Each $w^{j}$ contains an amplitude function $A_{1}^{j}$ which is a solution of the first order linear ordinary differential equation 


$$
A_{1}^{j}+\left(f^{\prime}(0)+\left(3 \lambda_{c} f^{\prime \prime \prime}(0) / 8\right) A_{1}^{1}(\tau)^{2}\right) A_{1}^{j}=R_{j}
$$

This equation is obtained from the orthogonality relation (21) with $k=j+2$. Then each $A_{1}^{j}(\tau)$ will be determined by an initial condition which follows from (20b) and (10). Now we find that as $\tau$ becomes infinite, the coefficient of each $A_{1}^{j}$ in (32) approaches the positive constant $-2 f^{\prime}(0)$, so that in the steady state each $A_{1}^{j}$ approaches a constant which depends only on the steady state value of $A_{1}^{1}(\tau)$. Thus, in the limit as $\tau$ becomes infinite, the entire expansion, and not just its leading term, describes a stationary state, and this stationary state is independent of the initial perturbation.

We further note that the stability of the new stationary solutions can be determined by this method, without recourse to an additional perturbation analysis. Thus from $(27)$ we see that for $\left|A_{1}^{1}(\tau)\right|$ less (greater) than its steady state value, $\dot{A}_{1}^{1}$ is positive (negative), so that $A_{1}^{1}$ will increase (decrease) to the stationary state. This is a statement of the stability of the stationary states. This result agrees with that of Millman and Keller [3], who first obtained the stationary states by a perturbation analysis, and then considered a linear stability analysis of those states.

It should be mentioned that in the model problem, $\lambda_{c}$ is a simple eigenvalue. It is for this reason that at each stage in the recursive determination of $w^{j}$, the orthogonality condition yields a single differential equation for the amplitude function $A_{1}^{j}$. In other problems, if the multiplicity of $\lambda_{c}$ is $M$, then at each stage of the recursive scheme, there will be $M$ orthogonality conditions for the determination of $M$ amplitude functions. Problems with multiple eigenvalues are considerably more difficult than those with simple eigenvalues. Finally, we mention that our method seems to enjoy certain advantages over existing methods. These are discussed in detail in [1].

The author is indebted to Professor L. Rubenfeld for a number of helpful discussions.

\section{REFERENCES}

1. B. J. Matkowsky, Nonlinear dynamic stability: A formal theory, SIAM J. Appl. Math. 18 (1970).

2. E. L. Reiss and B. J. Matkowsky, Nonlinear dynamic buckling of a compressed elastic column (to appear).

3. M. H. Millman and J. B. Keller, Perturbation theory of nonlinear boundary value problems, J. Mathematical Phys. 10 (1969), 342-361. MR 38 \#6146.

Rensselaer Polytechnic Institute, Troy, New York 12181 